\title{
Fertility Mapping of Soils from Hingoli and Sengaon Tahsils of Hingoli District, India
}

\author{
S.R. Adat*, T.R. Zagade and P.B. Chalawade \\ Department of Soil Science and Agril.Chemistry, Vasantrao Naik Marathwada \\ Krishi Vidyapeeth, Parbhani, India \\ *Corresponding author:
}

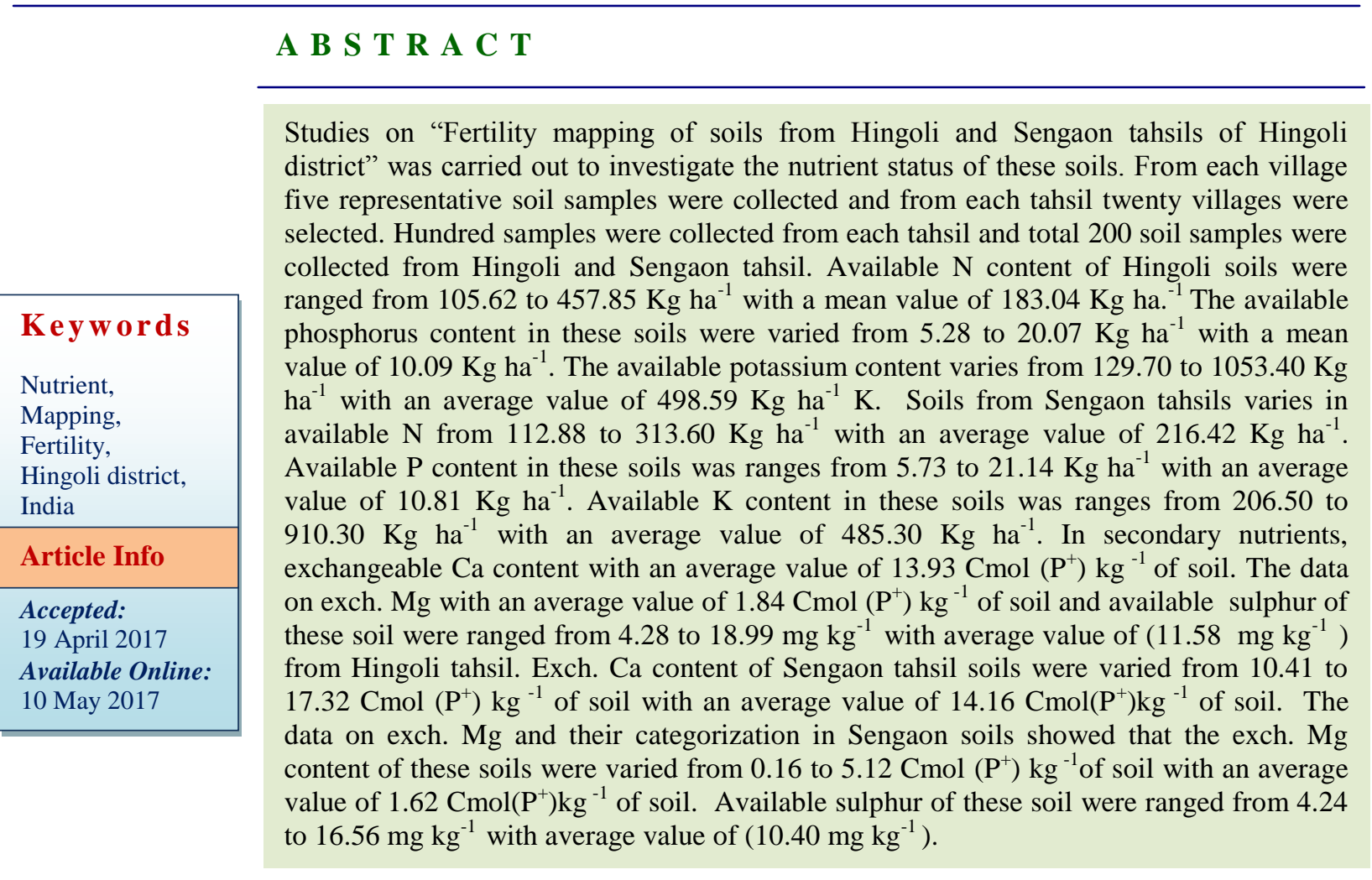

\section{Introduction}

Soils are a vital natural resource whose proper use greatly determines the capabilities of life support system and socio-economic development of people. Being important component of geosphere-biosphere system, Soil provides food, fiber, fodder, and fuel wood for varieties of basic human needs and shelter demand of future.
Therefore, management of soil resource on scientific principles is of prime important (Sarkar et al., 2002). Soil is a natural dynamic body containing mineral matter, organic matter and living forms considered to be store house of nutrients even though their continuous removal by intensive cropping. Among the several factors that influence crop 
production potential, soil fertility is fundamental factors. It is the integral part of soil and generally defined as capacity of soil to supply nutrient needed by crop in proper form and which having both direct and indirect effect on plant growth.

Intensive cropping and increased use of fertilizers, though there is increasing production tremendously, resulted in heavy turnover of nutrients from soil. There is a continuous decline in soil fertility and productivity due to exploitation of sol resource base. Imbalanced and indiscriminate use of fertilizers and emergence of micronutrients deficiencies have been identified as most important factors for declining crop productivity (Kanwar and Randhava, 1967) and Puri et al., (2003).

To know the present status of soil and future productivity, it is essential to know the fertility status. Considerable work on physicchemical properties of Maharastra soils was reported (Bharambe, 2001). Thus, it is necessary to define the areas of deficiency of particular nutrients in a particular areas and crops. Soil test data would be helpful in growing such deficient areas on soil and crop basis. Thus, the details of soil resource thematic maps and using data on various soil properties, focus given on fertility status, hence, present investigation is useful in judging the deficiencies of various nutrients.

\section{Materials and Methods}

Available macronutrient content in soils were determined by following methods.

\section{Available nitrogen}

Available nitrogen was determined by alkaline potassium permanganate method as suggested by (Subbiah and Asija, 1956).

\section{Available phosphorus}

The Available phosphorus was determined by Olsen's method using $0.5 \mathrm{M}$ sodium bicarbonate $\left(\mathrm{P}^{\mathrm{H}} 8.5\right)$ as an extracting reagent as described by (Chopra and Kanwar,1976).

\section{Available potassium}

The Available potassium was determined by soil treated with neutral normal ammonium acetate and the potassium in extract was determined by emission spectroscopy by flame photometer (Jackson,1967).

\section{Exchangeable Ca and Mg}

Exchangeable $\mathrm{Ca}$ and $\mathrm{Mg}$ was determined by using ammonium acetate extractant of soil by EDTA Versenate Method (Jackson, 1973)

\section{Available Sulphur}

The available sulphur was determined by using Turbidi metric method by using 1:5 soil and extractant $0.15 \% \mathrm{CaCl}_{2}$ solution on spectrophoto meter at $340 \mathrm{~nm}$ wavelength (William and Seinberes, 1969).

\section{Results and Discussion}

\section{Status of Available N, P, and K in soils}

The $\mathrm{N}, \mathrm{P}$ and $\mathrm{K}$ are the key nutrient, which are required for plant metabolism. Due to imbalance supply of faulty management practices there is decrease in availability of these nutrients. Hence wide spread deficiency or unavailability of nutrients in soils of Hingoli and Sengaon tahsils undertaking for study.

The data on status of $\mathrm{N}, \mathrm{P}$ and $\mathrm{K}$ and their categorization in soils of Hingoli tahsil showed in above table that the available $\mathrm{N}$ content of these soils were ranged from 
105.62 to $457.85 \mathrm{Kg} \mathrm{ha}^{-1}$ with a mean value of $183.04 \mathrm{Kg} \mathrm{ha} \mathrm{K}^{-1 .}$ The lowest $\mathrm{N}$ content $\left(105.62 \mathrm{Kg} \mathrm{ha}^{-1}\right)$ was observed in soils of Sakhara village whereas the highest $\mathrm{N}$ content $\left(457.85 \mathrm{Kg} \mathrm{ha}^{-1}\right)$ were recorded in soils of Bhankheda (Sample No. A96). Out of 20 villages the lowest range 118.15 to $187.80 \mathrm{Kg}$ $\mathrm{ha}^{-1}$ with an average value of $153.81 \mathrm{Kg} \mathrm{ha}^{-1}$ were recorded in Chaundi village while highest range 106.60 to $457.85 \mathrm{Kg} \mathrm{ha}^{-1}$ with a mean value of $211.95 \mathrm{Kg} \mathrm{ha}^{-1}$ was observed in Bhankheda. Out of 100 soil samples, 94 percent in low $\left(<250 \mathrm{Kg} \mathrm{ha}^{-1}\right)$ and 6 percent medium ( 250 to $500 \mathrm{Kg} \mathrm{ha}^{-1}$ ) in available $\mathrm{N}$ content.

The available phosphorus content in these soils were varied from 5.28 to $20.07 \mathrm{Kg} \mathrm{ha}^{-1}$ with a mean value of $10.09 \mathrm{Kg} \mathrm{ha}^{-1}$. The lowest P $\left(5.28 \mathrm{Kg} \mathrm{ha}^{-1}\right)$ content was observed in Sakhara village (Sample No. A9), while highest $\mathrm{P}\left(20.07 \mathrm{Kg} \mathrm{ha}^{-1}\right)$ was recorded in village Pimparilinga (Sample No. A55). These results clearly showed that the soils of Hingoli tahsil were low to medium in $\mathrm{P}$ content. The large range may be due to variation in soil properties and their high $\mathrm{P}$ fixing capacity, which prevents to come into readily available form in soil solution. These results were confirmity with Patil and Sonar, (1994) and Puri, (2009).

The available potassium contents in these soils were ranged from 129.70 to $1053.40 \mathrm{Kg}$ $\mathrm{ha}^{-1}$ with an average value of $498.59 \mathrm{Kg} \mathrm{ha}^{-1}$ $\mathrm{K}$. The lowest value of $\mathrm{K}$ was recorded in soils of Ghordari while highest value of $\mathrm{K}$ content was recorded in Suki village. Among twenty villages the lowest range in $\mathrm{K}$ content were ranges from 129.70 to $493.70 \mathrm{Kg} \mathrm{ha}^{-1}$ with an average value of $311.52 \mathrm{Kg} \mathrm{ha}^{-1}$ in village Ghordari while highest range 409.10 to $989.40 \mathrm{Kg} \mathrm{ha}^{-1}$ with an average value of $678.18 \mathrm{Kg} \mathrm{ha}^{-1} \mathrm{~K}$ were observed in Sakhara village. Out of 100 samples 3, 17 and 80 percent samples were categorized in low (<
$150 \mathrm{Kg} \mathrm{ha}^{-1}$ ), medium (150 to $300 \mathrm{Kg} \mathrm{ha}^{-1}$ ) and high (> $300 \mathrm{Kg} \mathrm{ha}^{-1}$ ) in $\mathrm{K}$ content respectively.

\section{Status of available N, P and K of Sengaon tahsil soils}

Available $\mathrm{N}$ content of these soils were varied from 112.88 to $313.60 \mathrm{Kg} \mathrm{ha}^{-1}$ with an average value of $216.42 \mathrm{Kg} \mathrm{ha}^{-1}$. The lowest available $\mathrm{N}$ content was recorded in village Bhankheda. While, highest content of $\mathrm{N}$ was observed in village Warudkazi. Further, data indicate that the lowest range of 118.15 to $187.80 \mathrm{Kg} \mathrm{ha}^{-1}$ with a mean value of 153.81 $\mathrm{Kg} \mathrm{ha}{ }^{-1}$ was observed in Chaundi village whereas, highest range 106.60 to $457.85 \mathrm{Kg}$ $\mathrm{ha}^{-1}$ were recorded with an average value of $211.95 \mathrm{Kg} \mathrm{ha}^{-1}$ in $\mathrm{N}$ content of these soils. Among twenty villages all villages were low in $\mathrm{N}$ content. Out of 100 soil samples 93 percent samples were low and 7 percent medium in available $\mathrm{N}$ content.

The available $\mathrm{P}$ content in these soils were ranges from 5.73 to $21.14 \mathrm{Kg} \mathrm{ha}^{-1}$ with an average value of $10.81 \mathrm{Kg} \mathrm{ha}^{-1}$. The lowest $\mathrm{P}$ content was recorded in village Pimparilinga whereas, highest $\mathrm{P}$ content was recorded in village Borada. The lowest range 5.73 to 9.58 $\mathrm{Kg} \mathrm{ha}^{-1}$ in available $\mathrm{P}$ content with a mean value of $7.66 \mathrm{Kg} \mathrm{ha}^{-1}$ were recorded in Pimparilinga village while highest range 10.57 to $21.14 \mathrm{Kg} \mathrm{ha}^{-1}$ with an average value of $15.62 \mathrm{Kg} \mathrm{ha}^{-1}$ were recorded in soils of Borala village. Among 20 villages, 10 villages were low and 10 villages which were medium in $\mathrm{P}$ content. Out of 100 soil samples 55 percent in low, and 45 per cent sample was categorized under medium content of available $P$.

The available $\mathrm{K}$ content in these soils were ranges from 206.50 to $910.30 \mathrm{Kg} \mathrm{ha}^{-1}$ with an average value of $485.30 \mathrm{Kg} \mathrm{ha}^{-1}$. The lowest value of $\mathrm{K}$ content was recorded in village 
Bhankheda whereas, highest value of $K$ content was observed in soils of Ganeshpur village. The lowest range 206.50 to 520.80 $\mathrm{Kg} \mathrm{ha}^{-1} \mathrm{~K}$ with mean value of $372.86 \mathrm{Kg} \mathrm{ha}^{-1}$ was recorded in Bhankheda village, while highest range 471.80 to $910.30 \mathrm{Kg} \mathrm{ha}^{-1}$ was observed in soils of Ganeshpur village. Out of Twenty villages, 13 villages viz., Bhankheda (372.86 Kg ha ${ }^{-1}$ ), Chaundi (469.72 Kg ha ${ }^{-1}$ ), Goregaon (406.22 $\mathrm{Kg} \mathrm{ha}^{-1}$ ), Gondala (406.96 $\left.\mathrm{Kg} \mathrm{ha}^{-1}\right)$, Ghordari (412.56 Kg ha $\left.{ }^{-1}\right)$, Kendre (495.74 Kg ha ${ }^{-1}$ ), Mhalsi (430.42 $\mathrm{Kg} \mathrm{ha}^{-1}$ ), Veltura (448.82 $\mathrm{Kg} \mathrm{ha}^{-1}$ ), Pimpaldari(452.00 $\mathrm{Kg} \mathrm{ha}^{-1}$ ), Vadhivra (479.84 $\mathrm{Kg} \mathrm{ha}^{-1}$ ) Sukli (493.18 Kg ha ${ }^{-1}$ ), Waghjali (399.50 Kg ha ${ }^{-1}$ ) and Warudkazi (459.86 $\left.\mathrm{Kg} \mathrm{ha}^{-1}\right)$ were categorized in medium $\mathrm{K}$ content and remaining villages were categorized under high content of $\mathrm{K}$.

Status of exchangeable $\mathrm{Ca}, \mathrm{Mg}$ and available Sulphur of Hingoli tahsil soils

Exch.Ca content of these soils were varied from 11.24 to $19.60 \mathrm{Cmol}\left(\mathrm{P}^{+}\right) \mathrm{kg}^{-1}$ of soil with an average value of $13.93 \mathrm{Cmol}\left(\mathrm{P}^{+}\right) \mathrm{kg}^{-1}$ of soil. The lowest available $\mathrm{Ca}$ content was recorded in village Sukli (Sample no.HQ1) While, highest content of $\mathrm{Ca}$ was observed in village Ajegaon (Soil sample No.HB2). Further, data indicate that the lowest range of 12.08 to $13.28 \mathrm{Cmol}\left(\mathrm{P}^{+}\right) \mathrm{kg}^{-1}$ of soil with a mean value of $12.82 \mathrm{Cmol}\left(\mathrm{P}^{+}\right) / \mathrm{kg}$ of soil observed in Hingoli village whereas, highest range 13.12 to $19.60 \mathrm{Cmol}\left(\mathrm{P}^{+}\right) \mathrm{kg}^{-1}$ of soil recorded with an average value of 14.95 $\mathrm{Cmol}\left(\mathrm{P}^{+}\right) / \mathrm{kg}$ of soil content of these soils. Out of 100 soil samples 91 per cent samples were low and 9 per cent medium in available $\mathrm{Ca}$ content. These value indicated that $\mathrm{Ca}$ content in the Hingoli soils were low to medium.

From above result, it was inferred that high calcium because of presence of $\mathrm{CaCO}_{3}$ which dominant source of calcium in soil and it liberate when mineral disintegrate and decompose. Mahapatra and Shahu (1996) reported that exchangable $\mathrm{Ca}^{++}$varied from 1.07 to $29.6 \mathrm{Cmol}\left(\mathrm{P}^{+}\right) \mathrm{kg}^{-1}$. Similar results were observed by More et al., (2002).

The data on exch. Mg and their categorization in Hingoli soils showed in revealed that the exch. $\mathrm{Mg}$ content of these soils were varied from 0.16 to $6.32 \mathrm{Cmol}\left(\mathrm{P}^{+}\right) \mathrm{kg}^{-1}$ of soil with an average value of $1.84 \mathrm{Cmol}\left(\mathrm{P}^{+}\right) \mathrm{kg}^{-1}$ of soil. The lowest available $\mathrm{Mg}$ content was recorded in village Kalkondi (sample no.HK5). While, highest content of $\mathrm{Mg}$ was observed in village Takli (sample no.HT3). Further, data indicate that the lowest range of 0.64 to $1.60 \mathrm{Cmol}\left(\mathrm{P}^{+}\right) \mathrm{kg}^{-1}$ of soil with a mean value of $1.28 \mathrm{Cmol}\left(\mathrm{P}^{+}\right) \mathrm{kg}^{-1}$ of soil observed in Karanjali village whereas, highest range 0.80 to $5.44 \mathrm{Cmol}\left(\mathrm{P}^{+}\right) \mathrm{kg}^{-1}$ of soil recorded with an average value of $2.92 \mathrm{Cmol}\left(\mathrm{P}^{+}\right) \mathrm{kg}^{-1}$ of soil of these soils. Out of 100 soil samples, 87 per cent samples were low, 10 percent medium and 3 per cent were low in available $\mathrm{Mg}$ content (Fig 5). Mahapatra and shahu,(1996) reported that exch. $\mathrm{Mg}^{+}$varied from 0.66 to $30.1 \mathrm{Cmol}\left(\mathrm{p}^{+}\right) \mathrm{kg}^{-1}$ Similar results quoted by More et al., (2002).

Available Sulphur of these soil were ranged from 4.28 to $18.99 \mathrm{mg} \mathrm{kg}^{-1}$ with average value of $\left(11.58 \mathrm{mg} \mathrm{kg}^{-1}\right)$. The lowest value $(4.28$ $\mathrm{mg} \mathrm{kg}^{-1}$ ) of $\mathrm{S}$ content was recorded in village Mauja, (sample no. A68) where as highest value of $S$ was recorded in soils of Digraj village. The lowest range 4.72 to $12.81 \mathrm{mg}$ $\mathrm{kg}^{-1}$ with mean value of $9.03 \mathrm{mg} \mathrm{kg}^{-1}$ were recorded in Boralwadi village while highest range 11.31 to $17.50 \mathrm{mg} \mathrm{kg}^{-1}$ with an average value of $14.44 \mathrm{mg} \mathrm{kg}^{-1}$ were observed in Ghota village in available $\mathrm{S}$ content in these soils of Hingoli tahsil.

\section{Status of exch. Ca, Mg and available $\mathrm{S}$ of Sengaon tahsil soils}

Exch. Ca content of these soils were varied from 10.41 to $17.32 \mathrm{Cmol}\left(\mathrm{P}^{+}\right) \mathrm{kg}^{-1}$ of soil with 
an average value of $14.16 \mathrm{Cmol}\left(\mathrm{P}^{+}\right) \mathrm{kg}^{-1}$ of soil. The lowest exch.Ca content was recorded in village Goregaon (sample no.SI2). While, highest content of $\mathrm{Ca}$ was observed in village Ajegaon (sample no.SB1 ). Further, data indicated that the lowest range of 10.41 to $15.12 \mathrm{Cmol}\left(\mathrm{P}^{+}\right) \mathrm{kg}^{-1}$ of soil with a mean value of $13.29 \mathrm{Cmol}\left(\mathrm{P}^{+}\right) \mathrm{kg}^{-1}$ of soil observed in Bhankheda village whereas, highest range 12.80 to $17.32 \mathrm{Cmol}\left(\mathrm{P}^{+}\right) \mathrm{kg}^{-1}$ of soil recorded with an average value of $14.85 \mathrm{Cmol}\left(\mathrm{P}^{+}\right) \mathrm{kg}^{-1}$ of soil content of these soils. Among twenty villages, maximum villages were low in $\mathrm{Ca}$ content. Out of 100 soil samples indicated that $\mathrm{Ca}$ content in the Sengaon soils were low to medium. From above result, it was inferred that high calcium because of presence of $\mathrm{CaCO}_{3}$ which dominant source of calcium in soil and it liberate when mineral disintegrate and decompose. The data on exch. $\mathrm{Mg}$ and their categorization in Sengaon soils showed in table 15 revealed that the exch. $\mathrm{Mg}$ content of these soils were varied from 0.16 to 5.12 $\mathrm{Cmol}\left(\mathrm{P}^{+}\right) \mathrm{kg}^{-1}$ of soil with an average value of $1.62 \mathrm{Cmol}\left(\mathrm{P}^{+}\right) \mathrm{kg}^{-1}$ of soil. The lowest available $\mathrm{Mg}$ content was recorded in village Mhalshi (Sample no.SK1). While, highest content of $\mathrm{Mg}$ was observed in village Ghordari (sample no.SG4).Among twenty villages, maximum villages were low in $\mathrm{Mg}$ content.

Table.1 Status of available N,P and K in soils of Hingoli tahsil from Hingoli distrct

\begin{tabular}{|c|c|c|c|c|}
\hline $\begin{array}{l}\text { Sr. } \\
\text { No. }\end{array}$ & $\begin{array}{c}\text { Sample } \\
\text { No. }\end{array}$ & $\begin{array}{c}\mathrm{N} \\
\left(\mathrm{Kg} \mathrm{ha}^{-1}\right)\end{array}$ & $\begin{array}{c}\mathrm{P} \\
\left(\mathrm{Kg} \mathrm{ha}^{-1}\right)\end{array}$ & $\begin{array}{c}\mathrm{K} \\
\left(\mathrm{Kg} \mathrm{ha}^{-1}\right)\end{array}$ \\
\hline 1 & HA1 & 185.45 & 12.00 & 311.10 \\
\hline 2 & HA2 & 126.78 & 9.84 & 381.20 \\
\hline 3 & HA3 & 180.45 & 15.90 & 237.40 \\
\hline 4 & HA4 & 194.43 & 11.91 & 403.70 \\
\hline 5 & HA5 & 240.60 & 10.03 & 341.50 \\
\hline 6 & HB1 & 214.24 & 8.96 & 139.00 \\
\hline 7 & HB2 & 244.60 & 11.51 & 493.40 \\
\hline 8 & HB3 & 195.22 & 7.43 & 894.30 \\
\hline 9 & HB4 & 165.20 & 11.91 & 367.80 \\
\hline 10 & HB5 & 147.34 & 8.06 & 797.90 \\
\hline 11 & $\mathrm{HC} 1$ & 158.45 & 10.43 & 471.80 \\
\hline 12 & $\mathrm{HC} 2$ & 457.85 & 9.58 & 424.90 \\
\hline 13 & $\mathrm{HC} 3$ & 210.10 & 8.96 & 335.80 \\
\hline 14 & $\mathrm{HC} 4$ & 126.78 & 6.98 & 813.60 \\
\hline 15 & HC5 & 106.60 & 6.76 & 308.20 \\
\hline 16 & HD1 & 187.80 & 9.40 & 385.40 \\
\hline 17 & HD2 & 154.66 & 10.57 & 287.50 \\
\hline 18 & HD3 & 142.28 & 9.77 & 645.00 \\
\hline 19 & HD4 & 166.20 & 12.00 & 367.80 \\
\hline 20 & HD5 & 118.15 & 6.09 & 273.60 \\
\hline 21 & HE1 & 380.40 & 19.26 & 368.90 \\
\hline 22 & HE2 & 125.40 & 7.51 & 558.00 \\
\hline 23 & HE3 & 185.72 & 6.63 & 242.30 \\
\hline 24 & HE4 & 106.68 & 7.17 & 526.10 \\
\hline 25 & HE5 & 188.42 & 17.20 & 669.30 \\
\hline 26 & HF1 & 179.90 & 8.51 & 652.70 \\
\hline 27 & HF2 & 178.75 & 9.04 & 337.00 \\
\hline
\end{tabular}


Int.J.Curr.Microbiol.App.Sci (2017) 6(5): 2227-2245

\begin{tabular}{|c|c|c|c|c|}
\hline 28 & HF3 & 191.20 & 6.89 & 486.40 \\
\hline 29 & HF4 & 174.39 & 17.90 & 451.60 \\
\hline 30 & HF5 & 142.12 & 9.98 & 571.20 \\
\hline 31 & HG1 & 129.64 & 5.82 & 296.50 \\
\hline 32 & HG2 & 196.42 & 14.51 & 1076.20 \\
\hline 33 & HG3 & 212.24 & 11.52 & 543.90 \\
\hline 34 & HG4 & 208.12 & 10.03 & 343.70 \\
\hline 35 & HG5 & 190.45 & 12.18 & 1486.90 \\
\hline 36 & HH1 & 219.42 & 8.96 & 316.20 \\
\hline 37 & $\mathrm{HH} 2$ & 176.38 & 6.23 & 129.70 \\
\hline 38 & HH3 & 144.28 & 7.57 & 493.70 \\
\hline 39 & $\mathrm{HH} 4$ & 195.56 & 10.00 & 168.80 \\
\hline 40 & HH5 & 212.44 & 7.97 & 449.20 \\
\hline 41 & HI1 & 210.79 & 11.24 & 506.80 \\
\hline 42 & HI2 & 238.33 & 14.24 & 349.80 \\
\hline 43 & HI3 & 181.88 & 11.64 & 446.60 \\
\hline 44 & HI4 & 150.98 & 8.24 & 282.70 \\
\hline 45 & HI5 & 225.78 & 16.93 & 588.50 \\
\hline 46 & HJ1 & 215.24 & 12.45 & 589.40 \\
\hline 47 & HJ2 & 175.32 & 10.57 & 438.30 \\
\hline 48 & HJ3 & 112.89 & 6.89 & 496.60 \\
\hline 49 & HJ4 & 210.11 & 9.22 & 416.00 \\
\hline 50 & HJ5 & 210.43 & 9.13 & 296.30 \\
\hline 51 & HK1 & 241.47 & 13.97 & 494.30 \\
\hline 52 & HK2 & 156.18 & 7.97 & 288.60 \\
\hline 53 & HK3 & 144.26 & 6.06 & $349 . .8$ \\
\hline 54 & HK4 & 215.12 & 6.89 & 626.70 \\
\hline 55 & HK5 & 133.76 & 7.43 & 756.60 \\
\hline 57 & HL2 & 135.68 & 8.24 & 651.50 \\
\hline
\end{tabular}


Int.J.Curr.Microbiol.App.Sci (2017) 6(5): 2227-2245

\begin{tabular}{|c|c|c|c|c|}
\hline 58 & HL3 & 173.38 & 7.70 & 281.00 \\
\hline 59 & HL4 & 192.14 & 13.44 & 253.10 \\
\hline 60 & HL5 & 150.52 & 8.24 & 423.10 \\
\hline 61 & HM1 & 144.32 & 7.43 & 401.80 \\
\hline 62 & HM2 & 187.85 & 6.63 & 609.10 \\
\hline 63 & HM3 & 128.08 & 10.03 & 200.50 \\
\hline 64 & HM4 & 180.42 & 9.63 & 304.80 \\
\hline 65 & HM5 & 166.25 & 10.30 & 694.40 \\
\hline 66 & HN1 & 238.33 & 5.28 & 512.10 \\
\hline 67 & HN2 & 105.62 & 7.16 & 409.10 \\
\hline 68 & HN3 & 290.51 & 15.59 & 864.50 \\
\hline 69 & HN4 & 140.82 & 11.91 & 615.80 \\
\hline 70 & HN5 & 195.88 & 17.90 & 989.40 \\
\hline 71 & HO1 & 168.75 & 11.37 & 210.30 \\
\hline 72 & $\mathrm{HO} 2$ & 270.52 & 12.27 & 329.60 \\
\hline 73 & $\mathrm{HO} 3$ & 174.5 & 6.90 & 636.20 \\
\hline 74 & $\mathrm{HO} 4$ & 158.24 & 9.67 & 485.10 \\
\hline 75 & HO5 & 172.48 & 10.43 & 388.30 \\
\hline 76 & HP1 & 177.52 & 10.04 & 283.30 \\
\hline 77 & HP2 & 128.30 & 6.89 & 228.70 \\
\hline 78 & HP3 & 180.32 & 11.46 & 494.70 \\
\hline 79 & HP4 & 195.84 & 11.91 & 396.50 \\
\hline 80 & HP5 & 182.45 & 11.10 & 456.40 \\
\hline 81 & HQ1 & 213.24 & 11.82 & 261.50 \\
\hline 82 & HQ2 & 125.78 & 7.30 & 510.50 \\
\hline 83 & HQ3 & 235.20 & 12.72 & 445.80 \\
\hline 84 & HQ4 & 260.28 & 15.85 & 390.80 \\
\hline 85 & HQ5 & 213.24 & 5.56 & 1053.40 \\
\hline 86 & HR1 & 166.20 & 8.37 & 250.30 \\
\hline
\end{tabular}




\begin{tabular}{|c|c|c|c|c|}
87 & HR2 & 125.74 & 6.36 & 671.10 \\
\hline 88 & HR3 & 189.28 & 11.11 & 401.80 \\
\hline 89 & HR4 & 194.43 & 6.09 & 225.70 \\
\hline 90 & HR5 & 166.22 & 14.51 & 376.80 \\
\hline 91 & HS1 & 191.29 & 10.57 & 800.40 \\
\hline 92 & HS2 & 187.85 & 9.31 & 693.40 \\
\hline 93 & HS3 & 146.28 & 7.43 & 737.20 \\
\hline 94 & HS4 & 123.30 & 6.72 & 367.50 \\
\hline 95 & HS5 & 153.82 & 7.88 & 385.40 \\
\hline 96 & HT1 & 185.45 & 14.16 & 275.10 \\
\hline 97 & HT2 & 188.00 & 12.19 & 348.60 \\
\hline 98 & HT3 & 193.36 & 11.20 & 270.00 \\
\hline 99 & HT4 & 124.12 & 9.13 & 670.60 \\
\hline 100 & HT5 & 215.88 & 12.27 & 580.80 \\
\hline Mean & & $\mathbf{1 8 3 . 1 0}$ & $\mathbf{1 0 . 2 0}$ & $\mathbf{4 6 3 . 4 5}$ \\
\hline
\end{tabular}

Table.2 N, P, and K status of Sengaon tahsil soils

\begin{tabular}{|c|c|c|c|c|}
\hline Sr.No. & $\begin{array}{c}\text { Sample } \\
\mathbf{N o .}\end{array}$ & $\begin{array}{c}\mathbf{N} \\
\left(\mathbf{K g ~ h a}^{-1}\right)\end{array}$ & $\begin{array}{c}\mathbf{P} \\
\left(\mathbf{K g ~ h a}^{-1}\right)\end{array}$ & $\begin{array}{c}\mathbf{K} \\
\left(\mathbf{K g ~ h a}^{-\mathbf{1}}\right)\end{array}$ \\
\hline 1 & SA1 & 188.10 & 14.11 & 629.80 \\
\hline 2 & SA2 & 132.68 & 8.51 & 787.60 \\
\hline 3 & SA3 & 165.80 & 6.89 & 424.10 \\
\hline 4 & SA4 & 154.18 & 8.24 & 445.20 \\
\hline 5 & SA5 & 140.02 & 7.88 & 256.90 \\
\hline 6 & SB1 & 216.38 & 7.97 & 906.80 \\
\hline 7 & SB2 & 205.16 & 7.61 & 550.80 \\
\hline 8 & SB3 & 185.02 & 9.22 & 446.80 \\
\hline 9 & SB4 & 222.65 & 11.37 & 270.70 \\
\hline 10 & SB5 & 310.40 & 7.52 & 550.50 \\
\hline 11 & SC1 & 150.54 & 8.15 & 450.80 \\
\hline 12 & SC2 & 256.60 & 14.15 & 253.40 \\
\hline 13 & SC3 & 112.88 & 10.57 & 520.80 \\
\hline 14 & SC4 & 182.75 & 7.62 & 432.80 \\
\hline 15 & SC5 & 191.83 & 6.09 & 206.50 \\
\hline 16 & SD1 & 206.97 & 16.93 & 337.60 \\
\hline 17 & SD2 & 197.00 & 9.36 & 605.20 \\
\hline 18 & SD3 & 219.52 & 14.78 & 507.60 \\
\hline 19 & SD4 & 191.29 & 18.45 & 426.40 \\
\hline
\end{tabular}


Int.J.Curr.Microbiol.App.Sci (2017) 6(5): 2227-2245

\begin{tabular}{|c|c|c|c|c|}
\hline 20 & SD5 & 142.62 & 10.12 & 471.80 \\
\hline 21 & SE1 & 263.42 & 12.45 & 366.50 \\
\hline 22 & SE2 & 205.10 & 10.93 & 509.20 \\
\hline 23 & SE3 & 194.48 & 12.18 & 325.80 \\
\hline 24 & SE4 & 200.70 & 7.17 & 233.00 \\
\hline 25 & SE5 & 184.65 & 11.64 & 596.60 \\
\hline 26 & SF1 & 238.18 & 16.57 & 277.20 \\
\hline 27 & SF2 & 206.10 & 6.98 & 375.80 \\
\hline 28 & SF3 & 200.70 & 10.03 & 635.80 \\
\hline 29 & SF4 & 156.75 & 13.97 & 325.80 \\
\hline 30 & SF5 & 242.80 & 12.85 & 420.20 \\
\hline 31 & SG1 & 163.20 & 9.04 & 910.30 \\
\hline 32 & $\mathrm{SG} 2$ & 170.85 & 15.05 & 650.30 \\
\hline 33 & SG3 & 144.25 & 7.52 & 475.20 \\
\hline 34 & SG4 & 159.93 & 8.96 & 628.50 \\
\hline 35 & SG5 & 181.88 & 6.45 & 171.80 \\
\hline 36 & SH1 & 124.30 & 9.14 & 378.50 \\
\hline 37 & $\mathrm{SH} 2$ & 176.15 & 9.58 & 268.30 \\
\hline 38 & SH3 & 163.84 & 15.41 & 562.40 \\
\hline 39 & SH4 & 198.65 & 13.96 & 327.80 \\
\hline 40 & SH5 & 172.82 & 16.53 & 430.20 \\
\hline 41 & SI1 & 188.16 & 13.88 & 420.00 \\
\hline 42 & SI2 & 175.88 & 9.67 & 270.40 \\
\hline 43 & SI3 & 192.12 & 7.16 & 734.00 \\
\hline 44 & SI4 & 191.29 & 9.76 & 737.20 \\
\hline 45 & SI5 & 169.52 & 6.45 & 317.10 \\
\hline 46 & SJ1 & 232.40 & 13.44 & 275.30 \\
\hline 47 & SJ2 & 185.02 & 10.57 & 737.20 \\
\hline 48 & SJ3 & 238.33 & 14.78 & 430.20 \\
\hline 49 & SJ4 & 313.60 & 21.14 & 712.90 \\
\hline 50 & SJ5 & 181.88 & 18.18 & 509.60 \\
\hline 51 & SK1 & 193.50 & 8.37 & 475.20 \\
\hline 52 & SK2 & 203.84 & 18.90 & 320.70 \\
\hline 53 & SK3 & 210.11 & 14.24 & 596.40 \\
\hline 54 & SK4 & 191.29 & 12.81 & 350.70 \\
\hline 55 & SK5 & 178.20 & 11.91 & 409.10 \\
\hline 56 & SL1 & 190.22 & 9.58 & 328.60 \\
\hline 57 & SL2 & 185.30 & 5.73 & 224.10 \\
\hline 58 & SL3 & 178.75 & 6.36 & 409.10 \\
\hline 59 & SL4 & 194.43 & 7.70 & 705.30 \\
\hline 60 & SL5 & 198.60 & 8.96 & 592.90 \\
\hline
\end{tabular}


Int.J.Curr.Microbiol.App.Sci (2017) 6(5): 2227-2245

\begin{tabular}{|c|c|c|c|c|}
\hline 61 & SM1 & 222.65 & 19.89 & 460.50 \\
\hline 62 & SM2 & 200.70 & 8.10 & 305.90 \\
\hline 63 & SM3 & 166.20 & 7.97 & 457.90 \\
\hline 64 & SM4 & 168.20 & 10.16 & 534.40 \\
\hline 65 & SM5 & 263.42 & 11.71 & 485.40 \\
\hline 66 & SN1 & 203.84 & 8.96 & 627.20 \\
\hline 67 & SN2 & 242.75 & 7.88 & 817.80 \\
\hline 68 & SN3 & 240.20 & 8.37 & 575.80 \\
\hline 69 & SN4 & 238.33 & 16.12 & 214.80 \\
\hline 70 & SN5 & 194.43 & 7.70 & 540.30 \\
\hline 71 & SO1 & 202.10 & 12.32 & 345.80 \\
\hline 72 & $\mathrm{SO} 2$ & 199.42 & 9.76 & 662.30 \\
\hline 73 & $\mathrm{SO} 3$ & 263.42 & 6.36 & 611.80 \\
\hline 74 & $\mathrm{SO} 4$ & 182.30 & 7.52 & 394.30 \\
\hline 75 & SO5 & 194.43 & 6.90 & 385.00 \\
\hline 76 & SP1 & 208.90 & 9.67 & 437.30 \\
\hline 77 & SP2 & 141.12 & 9.40 & 644.30 \\
\hline 78 & SP3 & 188.22 & 14.87 & 717.80 \\
\hline 79 & SP4 & 194.43 & 6.99 & 817.80 \\
\hline 80 & SP5 & 175.70 & 16.48 & 391.90 \\
\hline 81 & SQ1 & 182.48 & 9.31 & 510.20 \\
\hline 82 & SQ2 & 191.29 & 11.64 & 605.70 \\
\hline 83 & SQ3 & 165.60 & 14.69 & 220.60 \\
\hline 84 & SQ4 & 216.45 & 13.52 & 757.00 \\
\hline 85 & SQ5 & 170.24 & 10.93 & 372.40 \\
\hline 86 & SR1 & 241.47 & 8.37 & 415.30 \\
\hline 87 & SR2 & 197.56 & 7.16 & 220.30 \\
\hline 88 & SR3 & 214.38 & 11.12 & 637.80 \\
\hline 89 & SR4 & 188.72 & 7.61 & 315.40 \\
\hline 90 & SR5 & 435.90 & 12.09 & 408.70 \\
\hline 91 & SS1 & 222.65 & 10.03 & 509.60 \\
\hline 92 & SS2 & 248.40 & 7.03 & 306.30 \\
\hline 93 & SS3 & 172.10 & 8.96 & 366.20 \\
\hline 94 & SS4 & 185.30 & 7.70 & 720.80 \\
\hline 95 & SS5 & 291.52 & 12.00 & 528.80 \\
\hline 96 & ST1 & 178.75 & 13.61 & 625.60 \\
\hline 97 & ST2 & 275.96 & 12.36 & 437.40 \\
\hline 98 & ST3 & 140.34 & 6.45 & 602.40 \\
\hline 99 & ST4 & 205.10 & 6.98 & 395.20 \\
\hline 100 & ST5 & 313.60 & 21.13 & 238.70 \\
\hline Mean & & 216.42 & 10.81 & 485.3 \\
\hline
\end{tabular}


Table.3 Exchangable $\mathrm{Ca}, \mathrm{Mg}$ and available $\mathrm{S}$ status of soils

\begin{tabular}{|c|c|c|c|c|}
\hline Sr. No. & Sample No. & $\begin{array}{l}\mathrm{Ca} \text { Cmol } \\
\left(\mathbf{P}^{+}\right) \mathrm{kg}^{-1}\end{array}$ & $\begin{array}{c}\mathrm{Mg} \mathrm{Cmol} \\
\left(\mathbf{P}^{+}\right) \mathbf{~ k g}^{-1}\end{array}$ & $\underset{\mathrm{mg} \mathrm{kg}}{\mathrm{S}}$ \\
\hline 1 & HA1 & 12.88 & 2.40 & 08.80 \\
\hline 2 & HA2 & 13.60 & 0.80 & 5.74 \\
\hline 3 & HA3 & 14.00 & 5.44 & 12.84 \\
\hline 4 & HA4 & 13.04 & 1.28 & 13.99 \\
\hline 5 & HA5 & 13.36 & 4.72 & 9.20 \\
\hline 6 & HB1 & 14.00 & 1.76 & 11.73 \\
\hline 7 & HB2 & 19.60 & 5.12 & 8.50 \\
\hline 8 & HB3 & 14.08 & 2.00 & 4.72 \\
\hline 9 & HB4 & 13.96 & 1.32 & 7.42 \\
\hline 10 & HB5 & 13.12 & 1.76 & 12.81 \\
\hline 11 & $\mathrm{HC} 1$ & 14.24 & 1.12 & 7.36 \\
\hline 12 & $\mathrm{HC} 2$ & 16.00 & 0.88 & 13.61 \\
\hline 13 & HC3 & 13.84 & 4.80 & 11.63 \\
\hline 14 & $\mathrm{HC} 4$ & 14.08 & 1.60 & 8.07 \\
\hline 15 & HC5 & 13.76 & 1.76 & 11.97 \\
\hline 16 & HD1 & 11.24 & 6.32 & 670 \\
\hline 17 & HD2 & 15.36 & 1.44 & 12.88 \\
\hline 18 & HD3 & 14.72 & 2.16 & 9.38 \\
\hline 19 & HD4 & 14.24 & 1.48 & 7.05 \\
\hline 20 & HD5 & 14.72 & 1.20 & 10.55 \\
\hline 21 & HE1 & 14.32 & 1.68 & 11.31 \\
\hline 22 & HE2 & 17.20 & 1.24 & 17.22 \\
\hline 23 & HE3 & 16.24 & 0.56 & 14.09 \\
\hline 24 & HE4 & 12.80 & 1.28 & 12.08 \\
\hline 25 & HE5 & 14.00 & 2.48 & 17.50 \\
\hline 26 & HF1 & 12.08 & 1.36 & 14.965 \\
\hline
\end{tabular}


Int.J.Curr.Microbiol.App.Sci (2017) 6(5): 2227-2245

\begin{tabular}{|c|c|c|c|c|}
\hline 27 & HF2 & 13.76 & 2.88 & 12.631 \\
\hline 28 & HF3 & 13.52 & 0.88 & 14.930 \\
\hline 29 & HF4 & 14.32 & 0.88 & 4.28 \\
\hline 30 & HF5 & 13.60 & 2.00 & 7.81 \\
\hline 31 & HG1 & 12.40 & 2.72 & 11.97 \\
\hline 32 & HG2 & 14.60 & 1.20 & 9.12 \\
\hline 33 & HG3 & 18.00 & 0.89 & 7.26 \\
\hline 34 & HG4 & 13.64 & 1.48 & 14.06 \\
\hline 35 & HG5 & 10.28 & 2.24 & 16.21 \\
\hline 36 & HH1 & 12.92 & 1.56 & 12.56 \\
\hline 37 & $\mathrm{HH} 2$ & 13.28 & 0.80 & 15.97 \\
\hline 38 & $\mathrm{HH} 3$ & 12.80 & 2.56 & 12.46 \\
\hline 39 & HH4 & 12.08 & 0.87 & 11.45 \\
\hline 40 & HH5 & 15.04 & 1.00 & 11.18 \\
\hline 41 & HI1 & 12.48 & 1.28 & 14.79 \\
\hline 42 & HI2 & 11.36 & 1.44 & 8.65 \\
\hline 43 & HI3 & 14.56 & 1.12 & 11.73 \\
\hline 44 & HI4 & 17.48 & 1.24 & 6.84 \\
\hline 45 & HI5 & 13.64 & 1.88 & 9.02 \\
\hline 46 & HJ1 & 12.00 & 0.96 & 9.32 \\
\hline 47 & HJ2 & 13.92 & 2.24 & 7.63 \\
\hline 48 & HJ3 & 14.88 & 1.28 & 11.80 \\
\hline 49 & HJ4 & 12.04 & 2.96 & 12.67 \\
\hline 50 & HJ5 & 15.12 & 0.56 & 15.83 \\
\hline 51 & HK1 & 14.16 & 1.76 & 11.94 \\
\hline 52 & HK2 & 14.24 & 1.68 & 10.06 \\
\hline 53 & HK3 & 13.52 & 1.50 & 11.11 \\
\hline 54 & HK4 & 16.16 & 1.60 & 16.31 \\
\hline 55 & HK5 & 13.76 & 0.16 & 5.92 \\
\hline
\end{tabular}


Int.J.Curr.Microbiol.App.Sci (2017) 6(5): 2227-2245

\begin{tabular}{|c|c|c|c|c|}
\hline 56 & HL1 & 14.56 & 3.12 & 12.40 \\
\hline 57 & HL2 & 14.88 & 1.60 & 14.72 \\
\hline 58 & HL3 & 13.36 & 2.72 & 8.70 \\
\hline 59 & HL4 & 15.60 & 1.76 & 10.22 \\
\hline 60 & HL5 & 12.96 & 1.60 & 11.25 \\
\hline 61 & HM1 & 18.40 & 1.76 & 14.40 \\
\hline 62 & HM2 & 13.76 & 1.76 & 9.65 \\
\hline 63 & HM3 & 15.12 & 1.84 & 13.12 \\
\hline 64 & HM4 & 13.36 & 2.48 & 11.11 \\
\hline 65 & HM5 & 10.76 & 1.12 & 14.40 \\
\hline 66 & HN1 & 14.48 & 1.44 & 7.10 \\
\hline 67 & HN2 & 14.64 & 2.88 & 11.49 \\
\hline 68 & HN3 & 13.68 & 1.76 & 11.97 \\
\hline 69 & HN4 & 17.08 & 1.60 & 7.46 \\
\hline 70 & HN5 & 13.44 & 1.84 & 14.65 \\
\hline 71 & HO1 & 14.08 & 1.36 & 9.79 \\
\hline 72 & $\mathrm{HO} 2$ & 12.24 & 2.32 & 12.50 \\
\hline 73 & HO3 & 13.48 & 1.72 & 7.84 \\
\hline 74 & HO4 & 14.96 & 0.88 & 13.26 \\
\hline 75 & HO5 & 12.32 & 2.64 & 12.36 \\
\hline 76 & HP1 & 13.00 & 2.64 & 4.88 \\
\hline 77 & HP2 & 12.84 & 1.60 & 11.11 \\
\hline 78 & HP3 & 15.52 & 0.72 & 6.00 \\
\hline 79 & HP4 & 13.60 & 1.48 & 11.00 \\
\hline 80 & HP5 & 12.92 & 1.24 & 12.29 \\
\hline 81 & HQ1 & 11.24 & 2.16 & 10.72 \\
\hline 82 & HQ2 & 13.76 & 1.84 & 14.06 \\
\hline 83 & HQ3 & 14.82 & 2.70 & 18.99 \\
\hline 84 & HQ4 & 15.28 & 3.20 & 13.78 \\
\hline
\end{tabular}


Int.J.Curr.Microbiol.App.Sci (2017) 6(5): 2227-2245

\begin{tabular}{|c|c|c|c|c|}
\hline 85 & HQ5 & 13.62 & 1.92 & 12.80 \\
\hline 86 & HR1 & 14.88 & 0.64 & 13.75 \\
\hline 87 & HR2 & 11.24 & 1.60 & 12.98 \\
\hline 88 & HR3 & 13.52 & 1.44 & 9.25 \\
\hline 89 & HR4 & 13.60 & 1.16 & 6.98 \\
\hline 90 & HR5 & 12.48 & 1.56 & 14.75 \\
\hline 91 & HS1 & 15.04 & 0.89 & 14.93 \\
\hline 92 & HS2 & 13.92 & 1.68 & 9.02 \\
\hline 93 & HS3 & 16.36 & 1.68 & 9.20 \\
\hline 94 & HS4 & 13.76 & 0.85 & 13.19 \\
\hline 95 & HS5 & 13.32 & 1.80 & 13.81 \\
\hline 96 & HT1 & 13.88 & 1.08 & 11.73 \\
\hline 97 & HT2 & 14.00 & 1.54 & 14.93 \\
\hline 98 & HT3 & 14.48 & 6.32 & 11.73 \\
\hline 99 & HT4 & 13.84 & 1.96 & 5.62 \\
\hline 100 & HT5 & 14.68 & 0.36 & 11.01 \\
\hline & mean & $\mathbf{1 3 . 9 3}$ & $\mathbf{1 . 8 4}$ & $\mathbf{1 1 . 5 8}$ \\
\hline
\end{tabular}

Table.4 Exchangable $\mathrm{Ca}, \mathrm{Mg}$, and available $\mathrm{S}$ status of soils

\begin{tabular}{|c|l|c|c|c|}
\hline Sr.No. & $\begin{array}{c}\text { Sample } \\
\text { No. }\end{array}$ & $\begin{array}{c}\mathbf{C a ~ C m o l}_{-\mathbf{1}}\left(\mathbf{P}^{+}\right) \mathbf{k g} \\
\mathbf{M g} \mathbf{C m o l}\left(\mathbf{P}^{+}\right) \mathbf{k g}^{-}\end{array}$ & $\begin{array}{c}\text { Avail. S } \\
\left(\mathbf{~ m g ~ k g}^{-1}\right)\end{array}$ \\
\hline 1 & SA1 & 12.50 & 1.00 & 12.43 \\
\hline 2 & SA2 & 13.92 & 1.44 & 10.83 \\
\hline 3 & SA3 & 15.00 & 1.92 & 6.87 \\
\hline 4 & SA4 & 14.80 & 1.12 & 10.65 \\
\hline 5 & SA5 & 15.04 & 0.88 & 13.95 \\
\hline 6 & SB1 & 17.32 & 1.04 & 9.38 \\
\hline 7 & SB2 & 12.80 & 1.92 & 9.61 \\
\hline 8 & SB3 & 14.96 & 1.92 & 12.84 \\
\hline 9 & SB4 & 14.80 & 1.28 & 15.90 \\
\hline
\end{tabular}


Int.J.Curr.Microbiol.App.Sci (2017) 6(5): 2227-2245

\begin{tabular}{|c|c|c|c|c|}
\hline 10 & SB5 & 14.40 & 2.72 & 12.59 \\
\hline 11 & SC1 & 13.00 & 2.68 & 9.44 \\
\hline 12 & SC2 & 14.72 & 1.48 & 12.04 \\
\hline 13 & SC3 & 13.80 & 2.00 & 8.50 \\
\hline 14 & SC4 & 15.60 & 1.68 & 11.97 \\
\hline 15 & SC5 & 14.44 & 1.48 & 12.91 \\
\hline 16 & SD1 & 15.12 & 1.68 & 7.60 \\
\hline 17 & SD2 & 13.68 & 1.92 & 11.00 \\
\hline 18 & $\begin{array}{l}\text { SD3 } \\
\end{array}$ & 14.40 & 2.16 & 9.75 \\
\hline 19 & SD4 & 10.41 & 1.52 & 13.12 \\
\hline 20 & SD5 & 12.88 & 2.24 & 9.86 \\
\hline 21 & SE1 & 13.12 & 2.32 & 5.74 \\
\hline 22 & SE2 & 14.16 & 1.28 & 7.12 \\
\hline 23 & SE3 & 15.00 & 1.84 & 11.14 \\
\hline 24 & SE4 & 13.28 & 1.76 & 6.50 \\
\hline 25 & SE5 & 12.44 & 1.66 & 12.22 \\
\hline 26 & SF1 & 14.48 & 2.00 & 7.25 \\
\hline 27 & SF2 & 12.48 & 3.52 & 8.10 \\
\hline 28 & SF3 & 14.60 & 1.88 & 11.04 \\
\hline 29 & SF4 & 15.04 & 0.92 & 6.20 \\
\hline 30 & SF5 & 13.96 & 2.68 & 12.84 \\
\hline 31 & SG1 & 13.12 & 1.68 & 8.61 \\
\hline 32 & SG2 & 11.68 & 0.96 & 7.98 \\
\hline 33 & SG3 & 14.36 & 0.96 & 12.11 \\
\hline 34 & SG4 & 13.96 & 5.12 & 16.56 \\
\hline 35 & SG5 & 14.88 & 0.48 & 14.16 \\
\hline 36 & SH1 & 16.92 & 2.12 & 10.93 \\
\hline 37 & $\mathrm{SH} 2$ & 14.16 & 1.36 & 11.18 \\
\hline 38 & SH3 & 12.00 & 3.28 & 13.71 \\
\hline 39 & SH4 & 14.12 & 1.32 & 11.80 \\
\hline 40 & SH5 & 16.00 & 1.28 & 14.46 \\
\hline
\end{tabular}


Int.J.Curr.Microbiol.App.Sci (2017) 6(5): 2227-2245

\begin{tabular}{|c|c|c|c|c|}
\hline 41 & SI1 & 13.04 & 2.20 & 7.35 \\
\hline 42 & SI2 & 14.83 & 0.41 & 4.76 \\
\hline 43 & SI3 & 12.80 & 0.20 & 8.20 \\
\hline 44 & SI4 & 13.56 & 1.76 & 12.77 \\
\hline 45 & SI5 & 11.72 & 1.64 & 9.58 \\
\hline 46 & SJ1 & 14.37 & 1.37 & 7.35 \\
\hline 47 & SJ2 & 12.88 & 2.80 & 13.61 \\
\hline 48 & SJ3 & 15.28 & 2.32 & 8.24 \\
\hline 49 & SJ4 & 13.92 & 1.76 & 8.90 \\
\hline 50 & SJ5 & 14.96 & 1.92 & 7.80 \\
\hline 51 & SK1 & 14.88 & 0.16 & 5.72 \\
\hline 52 & SK2 & 15.64 & 1.64 & 8.68 \\
\hline 53 & SK3 & 11.56 & 1.32 & 5.79 \\
\hline 54 & SK4 & 13.84 & 0.52 & 4.24 \\
\hline 55 & SK5 & 14.08 & 1.64 & 9.36 \\
\hline 56 & SL1 & 11.36 & 2.00 & 7.40 \\
\hline 57 & SL2 & 16.88 & 0.64 & 5.70 \\
\hline 58 & SL3 & 13.28 & 2.00 & 11.98 \\
\hline 59 & SL4 & 14.36 & 1.60 & 9.65 \\
\hline 60 & SL5 & 16.16 & 3.08 & 14.53 \\
\hline 61 & SM1 & 14.00 & 1.68 & 11.80 \\
\hline 62 & SM2 & 14.56 & 1.84 & 10.13 \\
\hline 63 & SM3 & 13.00 & 1.28 & 4.65 \\
\hline 64 & SM4 & 18.36 & 1.52 & 12.53 \\
\hline 65 & SM5 & 14.60 & 1.48 & 8.61 \\
\hline 66 & SN1 & 13.04 & 2.40 & 13.47 \\
\hline 67 & SN2 & 16.40 & 1.68 & 8.88 \\
\hline 68 & SN3 & 13.36 & 1.84 & 10.06 \\
\hline 69 & SN4 & 14.72 & 0.64 & 14.75 \\
\hline 70 & SN5 & 19.16 & 1.72 & 9.82 \\
\hline 71 & SO1 & 14.08 & 1.56 & 10.65 \\
\hline
\end{tabular}


Int.J.Curr.Microbiol.App.Sci (2017) 6(5): 2227-2245

\begin{tabular}{|c|c|c|c|c|}
\hline 72 & $\mathrm{SO} 2$ & 13.48 & 1.64 & 8.60 \\
\hline 73 & SO3 & 14.44 & 1.04 & 9.30 \\
\hline 74 & SO4 & 11.28 & 1.44 & 11.49 \\
\hline 75 & SO5 & 13.92 & 1.96 & 11.52 \\
\hline 76 & SP1 & 14.92 & 1.76 & 8.40 \\
\hline 77 & SP2 & 14.12 & 1.24 & 13.57 \\
\hline 78 & SP3 & 12.48 & 1.96 & 4.74 \\
\hline 79 & SP4 & 11.96 & 1.60 & 12.32 \\
\hline 80 & SP5 & 15.04 & 1.44 & 9.47 \\
\hline 81 & SQ1 & 14.20 & 2.28 & 11.28 \\
\hline 82 & SQ2 & 14.64 & 0.52 & 9.30 \\
\hline 83 & SQ3 & 12.28 & 1.92 & 12.18 \\
\hline 84 & SQ4 & 13.84 & 1.52 & 12.63 \\
\hline 85 & SQ5 & 15.08 & 0.40 & 7.22 \\
\hline 86 & SR1 & 14.84 & 0.52 & 13.68 \\
\hline 87 & SR2 & 12.48 & 1.36 & 11.66 \\
\hline 88 & SR3 & 14.88 & 0.96 & 12.43 \\
\hline 89 & SR4 & 15.04 & 1.28 & 9.30 \\
\hline 90 & SR5 & 18.84 & 1.64 & 12.15 \\
\hline 91 & ss1 & 14.88 & 1.36 & 7.77 \\
\hline 92 & SS2 & 13.76 & 1.44 & 12.22 \\
\hline 93 & SS3 & 10.40 & 1.48 & 9.75 \\
\hline 94 & SS4 & 13.48 & 0.40 & 7.60 \\
\hline 95 & SS5 & 14.08 & 1.56 & 13.85 \\
\hline 96 & ST1 & 18.48 & 1.36 & 10.48 \\
\hline 97 & ST2 & 12.56 & 2.16 & 7.84 \\
\hline 98 & ST3 & 13.60 & 1.92 & 6.79 \\
\hline 99 & ST4 & 13.90 & 1.76 & 8.88 \\
\hline 100 & ST5 & 16.50 & 1.52 & 14.13 \\
\hline & MEAN & 14.10 & 1.63 & 10.40 \\
\hline
\end{tabular}


Available Sulphur of these soil were ranged from 4.24 to $16.56 \mathrm{mg} \mathrm{kg}^{-1}$ with average value of $\left(10.40 \mathrm{mg} \mathrm{kg}^{-1}\right)$. The lowest value $(4.24$ $\mathrm{mg} \mathrm{kg}^{-1}$ ) of $\mathrm{S}$ content was recorded in village Kalkondi, where as highest value of $\mathrm{S}$ was recorded in soils of Deulgaon village. The lowest range 4.24 to $9.36 \mathrm{mg} \mathrm{kg}^{-1}$ with mean value of $6.75 \mathrm{mg} \mathrm{kg}^{-1}$ were recorded in Kalkondi village while highest range 10.93 to $14.46 \mathrm{mg} \mathrm{kg}^{-1}$ with an average value of 12.41 $\mathrm{mg} \mathrm{kg}^{-1}$ were observed in Hingoli in available $\mathrm{S}$ content.

In conclusion, in all, from Hingoli tahsil 94 per cent soils were low and 6 per cent were placed in medium category in available $\mathrm{N}$ content whereas 92 per cent were low and 8 per cent were medium in availabl $\mathrm{N}$ content from Sengaon tahsil The Soils from Hingoli and Sengaon tahsil were found 54 per cent low and 46 per cent medium whereas from Sengaon 52 per cent in low and 48 per cent found in medium available $\mathrm{P}$ content. The available $\mathrm{K}$ content from Hingoli tahsil, 80 per cent soils were high, 17 per cent were medium K content and from Sengaon 88 per cent soils were high in $\mathrm{K}$ content.

The exch. Calcium and Magnesium in soils of Hingoli and Sengaon were varied from 10.28 to $19.60 \mathrm{Cmol}\left(\mathrm{P}^{+}\right) \mathrm{kg}^{-1}$ of soil and 10.19 to 19.16 $\mathrm{Cmol}\left(\mathrm{P}^{+}\right) \mathrm{kg}^{-1}$ of soil respectively. The soils from Hingoli and Sengaon tahsil were found high category in calcium content and low in magnesium. In case of available Sulphur, 67 per cent and 83 per cent were deficient in Hingoli and Sengaon tahsils, respectively. According to concept of "soil nutrient index", the status of available $\mathrm{N}$ and $\mathrm{P}$ are low to medium whereas $\mathrm{K}$ are high in soils of Hingoli and Sengaon tahsils. The exch. $\mathrm{Ca}$ is high and $\mathrm{Mg}$ is low in these soil whereas these soils are deficient in $\mathrm{S}$ content. Thus, it can be concluded that soils of Hingoli and Sengaon tahsil are low to medium in fertility status.

\section{References}

Aage, A.B; Magar S.M., Godhawale G.V. and Borgaonkar S.B (2007b). "Studies on available macronutrients status in Beed district of Maharashtra state." Int. J. of Tropical Agriculture., 25 (3).pp.487489.

Awasthi U. S.(2005). "Balanced nutrition:Need to address crucial issues". The Hindu survey of Indian Agriculture,pp. 3-12.

Babar S., Narkhede A.H., Rathod P.K., Rathod S.D. and Kamble B.M (2007). "Studies on forms of soil Potassium and their interrelationship in central and eastern Vidarbha region of Maharashtra, India". The Asian. J. of soil. Sci., 2 (1),pp.96-103.

Bharmbe P.R. and Ghonshikar C.P (1984). "Fertility status of soils in Jayakwadi command." J.maharastra agric. Univ.,9(3),pp.326-327.

Bhatnagar R.K., Bansal K.N. and Trivedi S.K (2003). "Distribution of Sulphur in some profiles of Shivpuri district of Madhya Pradesh." J. Indian. Soc. Soil Sci.,51(1),pp.74-76.

Binita N.K., Dasog G.S. and Patil P.L (2009). "Soil fertility mapping in Ghatprabha left bank canal command area of north Karnataka by geographic information system technique". Karnataka J. Agric Sci., 22 (1),pp.81-88.

Black, C.A (1965). " Methods of soil Analysis part-II". American society of Agronomy, In. C. Madison Wiscousin, USA.

Cate, R.B. and Nelson, L.A (1965). "Tech. Bull. Int. soil testing review”.,L, North Carding, USA.

Chopra,S.L. and Kanwar, J.B (1976). "Analytical Agricultural Chemistry". Kalyani publication., New Delhi.

Dhanya, V., Mathews., Patil,P.L. and Dasog, G.S (2009). "Identification of soil 
fertility constraints of a pilot site in coastal agro ecosystem of Karnataka by GIS techniques," Karnataka J. Agric. Sci., 22 (1),pp.77-80.

Dolui, A.K. and R. Bera (2001). "Soil tests for available Iron and their Relation with soil properties in some Alfisols of Orissa India". Int. J. of tropical agriculture., 19 (1-4),pp. 5-15.

Dwivedi,S.K.,Sharma,V.K and Bhardwaj,Vipin (2005). "Status of available nutrients in soils of cold arid region of Ladakh". J. Indian Soc. Soil Sci.,53(3),pp.421-423.

Gajbe, M.V., Londe, M.G. and Varade, S.B (1976)."Soils of Marathwada". J. Maharashtra Agric. Univ.1 (2-6),pp.5559.

Ghonsikar, C.P (1982). "Research Review Report on soils". Joint Agrisco
Maharashtra Agril. Univ. held at KKV, Dapoli.

Hegde D.M. and Sudhakara Bapu S.N (2001). "Nutrient management strategies in Agriculture", A future out look. Fertilizer News., 46 (12),pp.61-66.

Hundal, H.S., Rajkumar, Dhanwindar Singh, and Manchandra, J.S (2006). "Available nutrient and heavy metal status of soils of Punjab North West India". J. Indian Soc. Soil Sci. 54 (1),pp.50-56.

Jackson, M.L.(1967). "Soil chemical analysis", Prentice Hall of India pvt. Ltd, New Delhi. 46,pp.128-135 and 283.

Kanthaliya, P.C. and Bhatt, P.L (1991). "Relation between organic carbon and Available nutrients in some soils of sub humid zone". J. Indian Soc. Soil Sci. 39,pp.781-782.

\section{How to cite this article:}

Adat, S.R., T.R. Zagade and Chalawade, P.B. 2017. Fertility Mapping of Soils from Hingoli and Sengaon Tahsils of Hingoli District, India. Int.J.Curr.Microbiol.App.Sci. 6(5): 2227-2245. doi: https://doi.org/10.20546/ijcmas.2017.605.249 